\title{
Declaración del Comité Consultivo de Inmunizaciones de la Sociedad Chilena de Infectología acerca de la eficacia de la vacuna anti-neumocóccica 23 valente en el adulto mayor. Febrero 2010
}

\author{
Erna Ripoll M., M. Teresa Valenzuela B., Rodrigo Vergara F., Katia Abarca V., \\ Alma Muñoz M., Jorge Jiménez de la Jara, M. Eugenia Pinto C., Vivian Luchsinger F.
}

\section{3-Valent Pneumococcal Vaccine. Statement of the Consultive Committee of Immunizations on behalf of the Chilean Infectious Diseases Society. February 2010}

The article summarizes the scientific evidence related with protection conferred by the 23 valent polysacchardie vaccine against invasive pneumococcal disease, non bacteriemic pneumococcal pneumonia, and probable pneumococal pneumonia in the elderly. Elder patients with and without risk factors are considered. The impact of herd immunitty conferred by vaccination of children with conjugated pneumococcal vaccine in reduction of penumonia in adults is discussed. Based on this analysis, the Advisory Committee on Immunizations of the Chilean Infectious Disease Society makes recommendations on the most efficient vaccine strategy for reduction of pneumococcal pneumonia in the elderly.

Key words: 23 valent polysaccharide pneumococcal vaccine Streptococcus pneumoniae, pneumococcal invasive disease, pneumonia, elderly people.

Palabras clave: Vacuna anti-neumocóccica polisacárida 23-valente Streptococcus pneumoniae, enfermedad neumocóccica invasora, neumonías, adulto mayor.

\section{Introducción}

S treptococcus pneumoniae es uno de los principales patógenos humanos, responsable de patologías invasoras, como la enfermedad neumocóccica invasora (ENI), y de patologías no invasoras, tales como la neumonía no bacteriémica y la otitis media aguda. Afecta fundamentalmente a grupos de edades extremas, lactantes y adultos mayores, a las personas con co-morbilidades agregadas y a los infectados por el virus de la inmunodeficiencia humana (VIH), generando en todos ellos una alta letalidad.

En Estados Unidos de Norteamérica, S. pneumoniae es el responsable de más de 500.000 casos de neumonías, 50.000 casos de bacteriemia, 3.000 casos de meningitis y 40.000 muertes al año ${ }^{1,2}$. Es también el patógeno bacteriano que causa el mayor número de muertes en países en desarrollo.

La incidencia de enfermedad neumocóccica invasora en adultos inmunocompetentes es de 0,07 casos por 1.000 personas al año; sin embargo, en grupos de riesgo alcanza una incidencia de 23,4 casos por 1.000 al año ${ }^{3}$. En Chile, estudios de neumonía neumocóccica evidencian una letalidad intrahospitalaria de 13-20\% y niveles de resistencia de $S$. pneumoniae a penicilina de $9-15 \%{ }^{4,5}$.

La emergencia de cepas resistentes a antimicrobianos y los cambios actuales de los criterios de resistencia in vitro de $S$. pneumoniae a $\beta$-lactámicos, que deben considerar el sitio de la infección según el Clinical and Laboratory Standards Institute (CLSI) ${ }^{6}$, son un gran desafío y complican el entendimiento de las actuales recomendaciones para el tratamiento de esta enfermedad, por lo que el énfasis debe situarse en la prevención. Así, para la infección meníngea se considera que $S$. pneumoniae es resistente a penicilina si la concentración inhibitoria mínima (CIM) es igual o superior a $0,12 \mu \mathrm{g} / \mathrm{ml}$. En el caso de localización no meníngea, que incluye neumonía bacteriémica y no bacteriémica, la cepa es considerada resistente si la CIM es igual o superior a $8 \mu \mathrm{g} / \mathrm{ml}^{6}$.

Si bien frente a este agente infeccioso existe una vacuna indicada para el adulto y niños sobre dos años de edad, en base a polisacárido capsular 23 valente, los resultados de la efectividad han sido controversiales. Respecto a estas discusiones y considerando la reciente incorporación de esta vacuna en el Programa Nacional de Inmunizaciones, el Comité Consultivo de Inmunizaciones (CCI) de la Sociedad Chilena de Infectología
Departamento de Enfermedades Respiratorias y Alérgicas, CENTROMED, Viña del Mar (ERM): Departamento de Salud Pública y Epidemiología, Facultad de Medicina, Universidad de los Andes (MTVB); Escuela de Medicina Universidad de Valparaíso y Servicio de Pediatría Hospital Carlos van Buren, Valparaíso (RVF): Departamento de Pediatría, Facultad de Medicina Pontificia Universidad Católica de Chile (KAV); Centro para Vacunas en Desarrollo-Chile (AMM); Escuela de Salud Pública, Facultad de Medicina Pontificia Universidad Católica de Chile (JDU): Laboratorio Clínico, Hospital Clínico, Universidad de Chile (MEPC); Programa de Virología ICBM, Facultad de Medicina, Universidad de Chile (VLF).

Correspondencia a: Erna Ripoll Moraga ernaripoll@hotmail.com 
(SOCHINF) se ha planteado como propósito revisar los estudios, los diseños y sus resultados de eficacia frente a ENI, neumonías neumocóccicas probables y confirmadas, para posteriormente formular una declaración frente a dicha vacuna.

\section{Vacuna neumocóccica polisacárida}

\section{Generalidades}

Se han descrito en la actualidad más de 90 serotipos de $S$. pneumoniae ${ }^{1}$. Los relacionados con enfermedad varían según la localización geográfica; sin embargo, 20 son los serotipos responsables de $75 \%$ de las infecciones invasoras en cualquier grupo de edad.

El desarrollo de la vacuna neumocóccica se inició en 1914 con Wright en Sudáfrica quien vacunó a mineros del oro con una suspensión de $S$. pneumoniae muertos y obtuvo un nivel de protección frente a la neumonía de $\sim 60-80 \% 0^{7,8}$.

La vacuna neumocóccica en uso para el adulto desde 1980, cubre 23 serotipos, responsables de más de $90 \%$ de las ENI en el adulto mayor. Actualmente existen tres laboratorios productores de estas vacunas: Sanofi (Pneumo -23 ${ }^{\circledR}$ ), Merck (Pneumovax 23 ${ }^{\circledR}$ ) y Lederle (Pnu-Immune $23^{\circledR}$ ).

Los serotipos contenidos en la vacuna son: 1, 2, 3, 4, $5,6 \mathrm{~B}, 7 \mathrm{~F}, 8,9 \mathrm{~N}, 9 \mathrm{~V}, 10 \mathrm{~A}, 11 \mathrm{~A}, 12 \mathrm{~F}, 14,15 \mathrm{~B}, 17 \mathrm{~F}, 18 \mathrm{C}$, 19A, 19F, 20, 22F, 23F y $33 \mathrm{~F}^{1}$. Entre estos serotipos se encuentran aquellos más asociados con la enfermedad invasora $^{2}$ y con resistencia a penicilina.

La dosis de $0,5 \mathrm{ml}$ de la vacuna contiene 25 microgramos de polisacárido capsular purificado, disuelto en solución salina con fenol o timerosal, sin adyuvante. Se administra en una dosis única, vía intramuscular o subcutánea. Existen situaciones clínicas de excepción que requieren recibir más de una dosis, tales como pacientes esplenectomizados o asplénicos.

\section{Inmunogenicidad}

La respuesta inmune a los antígenos de la vacuna es T-independiente, por lo que es pobremente inmunogénica en niños bajo dos años de edad y no induce inmunidad de memoria. A partir de los dos años de edad, induce un buen desarrollo de anticuerpos, edad y serotipo dependiente, aunque el nivel con el cual se obtiene protección aún no está establecido, como tampoco la duración de ella. No genera protección a nivel de mucosas ${ }^{1,9}$.

En un estudio chileno se pudo demostrar la permanencia de los niveles de anticuerpos al cabo de dos años de seguimiento en adultos mayores de 60 años de edad ${ }^{10}$. La relación entre concentración sérica de anticuerpos y protección otorgada por la vacuna polisacárida no ha sido bien establecida ${ }^{3,27}$.

\section{Seguridad}

La vacuna polisacárida ha demostrado ser segura con escasos reportes de eventos adversos asociados a ella. Las reacciones locales en general son leves, tales como enrojecimiento y dolor transitorio en el sitio de la punción. Se estima que un tercio de las personas vacunadas desarrollan los síntomas y signos descritos. Estas reacciones generalmente persisten por menos de 48 horas. Las reacciones moderadas sistémicas -fiebre y mialgias-y las locales de mayor gravedad -induración local-son escasas.

Las re-vacunaciones con este producto, con intervalos de tiempo superiores a cuatro años, no se asocian con un aumento de eventos adversos. Sólo en revacunaciones en períodos más cortos se ha observado un incremento de eventos adversos moderados 9 .

No se recomienda su uso durante el embarazo, pero no se han reportado problemas con la vacunación inadvertida en este periodo.

\section{Eficacia clínica de la vacuna polisacárida}

A pesar de múltiples estudios realizados por casi 30 años con la vacuna polisacárida, los resultados de su eficacia aún son controvertidos. Las razones son varias: utilización de diferentes vacunas, distintos diseños de estudios epidemiológicos, escasos estudios clínicos controlados, randomizados, doble ciego, falta de descripción de los métodos de enrolamiento de los sujetos al estudio, inclusión de estudios con falta de definición de las poblaciones a estudiar en cuanto a procedencia y grupos de edades bien definidos, falta de estandarización de los criterios de diagnóstico de neumonía y neumonía neumocóccica, entre otras.

En los últimos años se han publicado siete metaanálisis $^{11-17}$, dos revisiones sistemáticas de la base de datos Cochrane ${ }^{18,22}$ y dos documentos de la Organización Mundial de la Salud (OMS) ${ }^{9,23}$ refiriéndose a este tópico.

Los meta-análisis demuestran protección contra la ENI en el adulto mayor, aunque esta eficacia entre los subgrupos de adultos con enfermedades crónicas es escasa en comparación con la alcanzada en adultos sanos. Este efecto resulta ser paradójico dado que los grupos de mayor riesgo de ENI en el adulto mayor son los pacientes con patologías concomitantes, tales como diabetes mellitus, cáncer, enfermedades hematológicas, entre otras; el riesgo de ENI en adultos sanos entre 65 y 79 años de edad es del orden de 23 por 100.000 habitantes, el que aumenta a 460 por 100.000 habitantes en pacientes con cáncer ${ }^{19}$.

Respecto del rol protector de la vacuna neumocóccica polisacárida para neumonías neumocóccicas no bacteriémicas, ni los estudios clínicos experimentales ${ }^{20}$ ni los estudios epidemiológicos observacionales sustentan la eficacia de la vacuna ${ }^{24-29}$.

El último meta-análisis publicado en el año $2009^{16}$ concluye hallazgos similares a los anteriores: muestra 
un riesgo relativo (RR) combinado para la bacteriemia de 0,90 (IC 95\% 0,46-1,77) y de 0,62 (IC 95\% 0,05$8,61)$ para las neumonías neumocóccicas. Por otra parte, cuando el análisis se estratifica por calidad metodológica de los estudios clínicos de alta calidad (doble ciego o con diseño aleatorio adecuado) presentan un RR de 1,20 (IC 95\% 0,75-1,92) para las neumonías presuntivamente neumocóccicas. La eficacia contra todas las causas de neumonía no es concluyente, con una heterogeneidad estadística sustancial RR 0,89 (IC 95\% 0,69-1,14)2.

Por otro lado, desde la introducción de la vacuna neumocóccica conjugada 7 valente en niños a contar del año 2000, en E.U.A., se han observado interesantes cambios en la epidemiología de la ENI en los adultos, atribuibles al efecto de rebaño de la vacuna ${ }^{33-35}$. Las tasas de ENI debida a los serotipos contenidos en la vacuna 7-valente, en la población adulta (95\% IC) han disminuido desde 22,4 casos por 100.000 por año entre $1998-1999$ a 10,2 casos por 100.000 habitantes al año $2004^{33}$. Los únicos serotipos que han tenido un aumento como causantes de enfermedad invasora son el 3 y el $19 \mathrm{~A}^{33,34}$. El efecto global en la incidencia de ENI en el adulto mayor ha sido una disminución de 40,8 casos por 100.000 habitantes en el año 1998 a 29,4 casos por 100.000 habitantes en el año $2003^{33}$. Hoy se cuenta con vacunas conjugadas con $10 \mathrm{y}$ 13 serotipos cuyo uso en forma programática permitiría esperar un mayor impacto que el obtenido con la vacuna 7 valente.

\section{Discusión}

Los meta-análisis publicados a la fecha sobre la eficacia de la vacuna neumocóccica polisacárida 23-valente, incluyen escasos trabajos en población homologable a la chilena, puesto que han sido realizados especialmente en países europeos y norteamericanos. En algunos de ellos, los criterios de inclusión han sido amplios en cuanto a rangos de edades, por lo que los análisis no sólo se centran en la eficacia de ella en el adulto mayor.

En el meta-análisis de Melegaro $^{15}$ se compararon estudios de eficacia realizados en adultos mayores, tres de los cuales fueron en población norteamericana ${ }^{24-25}$. Los resultados globales de eficacia de la vacuna, expresada como $\mathrm{EV}=(1-\mathrm{OR}) * 100(\%)$ en pacientes con neumonía neumocóccica, sin factores de riesgo, variaron desde - $28 \%$ (-153\% - 35\% [95\% IC]) a 76\% (-150\% a 98\%). Al estimar por efectos aleatorios, la eficacia sigue siendo baja, $16 \%$ $(-50 \%$ a $53 \%)$. La eficacia estudiada en pacientes con neumonía neumocóccica con factores de riesgo fue de $-20 \%(-92 \% \text { a } 25 \%)^{15}$.

En este mismo meta-análisis la eficacia de la vacuna contra ENI fue de $65 \%$ (-49\% a 92\%). El análisis de la eficacia contra esta entidad clínica en pacientes con factores de riesgo es de $20 \%$ (-187\% a $78 \%$ ).
Otro meta-análisis comparó los resultados de la eficacia entre estudios de caso control, de cohorte y randomizados de cohorte con asignación aleatoria en el adulto mayor ${ }^{17}$. La eficacia encontrada de la vacuna polisacárida para ENI en los estudios observacionales fue de 55\% (OR: 0,53 $95 \%$ IC 0,35-0,81), su calidad se estimó como moderada, y los resultados fueron consistentes y homogéneos. La información de estos estudios referente a la protección para neumonía fue menor por problemas importantes en la comparación entre vacunados y no vacunados: los resultados fueron heterogéneos e inconsistentes y no mostraron eficacia de la vacuna para proteger contra neumonía al adulto mayor.

El último meta-análisis publicado a la fecha corresponde al de Huss, $2009^{16}$, el que incluye estudios abiertos, controlados, doble ciego, no solamente acotados al adulto mayor.

Los resultados de los principales meta-análisis se observan en la Tabla 1.

Una revisión sistemática de la base de datos Cochra$n \mathrm{e}^{22}$ sobre la efectividad de la vacuna polisacárida para prevenir enfermedad o muerte en adultos establece que la vacuna no es capaz de evidenciar la reducción de riesgo de neumonía o muerte por neumonía neumocóccica en adultos sobre 55 años de edad con enfermedades crónicas, pero podría ser capaz de reducir la ENI.

Tampoco la vacuna polisacárida es capaz de disminuir la portación nasofaríngea de serotipos contenidos en la vacuna $\mathrm{y}$, por ende, no actuará en la reducción de la transmisión del agente a la población ${ }^{23}$.

Los estudios que presentan los mejores resultados en términos de eficacia de la vacuna son los epidemiológicos de tipo observacional ${ }^{27-31}$, otorgándole a la vacuna un nivel de protección para bacteriemia de $50-80 \%$ en adultos inmunocompetentes.

\section{Conclusiones}

La efectividad de la vacuna polisacárida en el adulto mayor, licenciada desde hace más de 30 años atrás, ha sido cuestionada en los últimos años, debido a que ha sido difícil comparar lo logrado en poblaciones altamente heterogéneas usando distintos tipos de diseños de estudios, con diferentes criterios de inclusión y de definición de caso y con diferentes valencias de la vacuna; algunos de ellos han utilizado la vacuna 14 valente y otros la 23 valente. Los distintos resultados en los niveles de protección de la vacuna pueden estar dados por las características del hospedero, la epidemiología de la población en estudio y el diseño de los estudios, entre otros.

Las actuales recomendaciones de vacunación están fundamentadas en estudios observacionales, como los de caso-control y observacionales. 
Tabla 1. Resumen de meta-análisis sobre la efectividad de la vacuna polisacárida 23-valente contra Streptococcus pneumoniae en el adulto mayor

\begin{tabular}{|c|c|c|c|c|c|}
\hline \multirow{2}{*}{\multicolumn{2}{|c|}{ Estudio }} & \multicolumn{2}{|c|}{ Neumonía neumocóccica } & \multicolumn{2}{|c|}{ Bacteriemia } \\
\hline & & $\begin{array}{l}\text { Sin factores de riesgo } \\
\text { (IC } 95 \%)\end{array}$ & $\begin{array}{c}\text { Con factores de riesgo } \\
\text { (IC 95\%) }\end{array}$ & $\begin{array}{c}\text { Sin factores de riesgo } \\
\text { (IC } 95 \%)\end{array}$ & $\begin{array}{c}\text { Con factores de riesgo } \\
\text { (IC 95\%) }\end{array}$ \\
\hline Hutchinson & 1999 & OR $0,58(0,47-0,72)$ & OR $1,15(0,36-3,74)$ & OR $0,27(0,13-0,49)$ & No disponible \\
\hline Cornu & 2001 & OR 0,29 $(0,20-0,42)$ & OR $0,58(0,18-1,00)$ & No disponible & No disponible \\
\hline Watson & 2002 & OR 0,81 $(0,49-1,33)$ & OR $1,27(0,65-2,49)$ & OR $0,37(0,07-1,91)$ & No disponible \\
\hline Melegaro & 2004 & OR $0,84(0,47-1,50)$ & OR $1,20(0,75-1,92)$ & OR $0,35(0,08-1,49)$ & OR $0,80(0,22-2,88)$ \\
\hline Conaty & 2004 & \multicolumn{2}{|c|}{ OR $1,03 \quad(0,86-1,25)$} & \multicolumn{2}{|c|}{ OR 0,51 $(0,21-1,23)$} \\
\hline Huss & 2009 & \multicolumn{2}{|c|}{ RR: $1,04 \quad(0,78-1,38)$} & \multicolumn{2}{|c|}{ No disponible } \\
\hline
\end{tabular}

Los meta-análisis que analizan los estudios controlados en adultos mayores no muestran eficacia de la vacuna para protegerlos contra la neumonía neumocóccica.

La crítica que se hace a estos resultados se fundamenta en que los estudios observacionales son realizados con un número significativo de personas, reflejando así en mejor forma lo que sucede en la realidad, pero con sesgos interesantes de abordar. No así los estudios controlados en los que la población en estudio involucra un menor número de participantes ya que deben reunir ciertos criterios de inclusión y exclusión, con un menor sesgo y una mejor distribución aleatoria entre los grupos.

En todo caso parece razonable no incluir esta vacuna dentro de un programa gubernamental hasta no tener información con un mayor nivel de evidencia en cuanto a la protección que puede conferir al adulto mayor frente a la neumonía neumocóccica.

La evidencia existente a la fecha muestra resultados promisorios de impacto en la reducción de la ENI en el adulto mayor, gracias al efecto de rebaño otorgado por la vacunación universal del lactante mediante la vacuna neumocóccica conjugada.

Por todo lo expuesto el CCI formula las siguientes recomendaciones con respecto a la vacunación contra $S$. pneumoniae en el adulto en Chile:

- Los datos disponibles a la fecha a través de los estudios de mayor calidad, tales como los ensayos clínicos controlados y randomizados (ECCR), no son consistentes en demostrar la eficacia de la vacuna 23 valente contra neumonías en el adulto mayor, menos aún en quienes padecen de patologías de base o son inmunosuprimidos.

- Un programa que emplee la vacuna polisacárida no logrará disminuir la portación del agente ni efecto de rebaño en los contactos de la población vacunada.

- En países con recursos limitados para sustentar los programas de inmunización no es recomendable derivarlos hacia la vacuna polisacárida por la falta de evidencia en su rol protector contra las neumonías, especialmente en aquellos adultos mayores con algún grado de inmunocompromiso.

- Dado que la vacunación universal del lactante, mediante vacunas conjugadas, ha demostrado un importante efecto de rebaño en las distintas edades, más aún en el adulto mayor, la prioridad debiera centrarse en el uso de ellas.

\section{Resumen}

El artículo resume los hallazgos de la evidencia científica en relación a la protección que confiere la vacuna neumocóccica polisacárida 23 valente contra la enfermedad neumocóccica invasora, las neumonías neumocóccicas no bacteriémicas y las neumonías probablemente de etiología neumocóccica en el adulto mayor, estableciendo una categorización entre adultos mayores con factores de riesgo y sin ellos. Se documenta a la vez, el impacto que ha tenido la vacuna neumocóccica conjugada en la población infantil, en reducir las neumonías en el adulto mayor. Basados en la información disponible, el Comité Consultivo de Inmunizaciones de la Sociedad Chilena de Infectología, realiza recomendaciones sobre cuál sería la mejor forma de prevenir las enfermedades neumocóccicas en el adulto mayor.

\section{Potenciales conflictos de interés:}

Erna Ripoll M no tiene conflictos de interés con esta vacuna. Ha asistido a congresos financiada por GlaxoSmithKline y Merck.

M. Teresa Valenzuela B, ha participado en comité de expertos en vacunas antineumocóccicas de Wyeth.

Rodrigo Vergara F: no tiene conflictos de interés con esta vacuna.

Ha asistido a congresos y realizado estudios financiados por GlaxoSmithKline.

Katia Abarca V. ha recibido financiamiento de Sanofi 
Pasteur y Glaxo-SmithKline para estudios clínicos de vacunas; ha asistido a congresos y cursos auspiciados por Sanofi Pasteur, Merck, GlaxoSmithKline y Wyeth; ha recibido honorarios por participar en comité de expertos en vacunas de GlaxoSmithKline y Wyeth.

Alma Muñoz M. ha participado como investigador prin- cipal o sub investigador en estudios clínicos financiados por GlaxoSmithKline, Sanofi-Pasteur, Novartis, Medimmune. Además, en una oportunidad, Berna financió la asistencia a un congreso en el exterior.

Jorge Jiménez de la J., Vivian Luchsinger F., M. Eugenia

Pinto C. no tienen conflictos de interés.

\section{Referencias}

1.- Shorr A. Preventing pneumonia The role for pneumococcal and influenza vaccines. Clin Chest Med 2005; 26: 123-34.

2.- Prevención de enfermedad neumocócica: recomendaciones del Comité de Inmunizaciones Americano (Advisory Committee on Immunization Practice, ACIP) Morbid Mortal Wkly Rep MMWR Comm Rep 1997; 46 (RR-8): 1-24.

3.- Gordon S, French N. Should we develop an inhaled anti-peumococcal vaccine for adults? Curr Med Chem 2005; 4: 75-89.

4.- Rioseco M. Neumonía neumocóccica en 45 adultos hospitalizados. Rev Méd Chile 2004; 132: 588-94

5.- Díaz A, Torres C, Flores L, García P, Saldías F. Neumonía neumocóccica adquirida en la comunidad en adultos hospitalizados. Rev Méd Chile 2003; 131: 505-14.

6.- Clinical and Laboratory Standards Institute. Performance Standards for Antimicrobial Susceptibility Testing; Nineteenth Informational Supplement 2009; 28 (1).

7.- $\quad$ Smit P, Oberholzer D, Hayden- Smith S, Koornhof H J, Hillerman M R. Protective efficacy of pneumococcal polysacharide vaccines. JAMA 1977; 238: 2613-6.

8.- Austrian R, Douglas R M, Schiffman G. Prevention of pneumococcal pneumonia by vaccination. Trans Assoc Am Physicians 1976; 89: 184-94.

9.- WHO position paper. 23-valent pneumococcal polysaccharide vaccine. Wkly Epidemiol Rec No. 42, 17 October 2008, pp 373-84.

10.- Valenzuela M T, Altuzarra R, Trucco O, Villegas R, Inostroza J, Granata P, et al. Immunogenicity of a 23-valent pneumococcal polysaccharide vaccine in elderly residents of a long-term care facility. BJID 2007; 11 (3): 322-6.

11.- Hutchison B, Oxman A, Shannon H, Loyd S, Altmayer C, Thomas K. Clinical effectiveness of pneumococcal vaccine. Can Fam Physician 1999; 45: 2381-93.

12.- Cornu C, Yzebe D, Leophone P, Gaillat J, Boissel J P, Cucherat M. Efficacy of pneumococcal polysaccharide vaccine in immunocompetent adults: a meta-analysis of randomized trials. Vaccine 2001; 19 (32): 4780-90.

13.- Watson L, Wilson B, Waugh N. Pneumococcal polysaccharide vaccine: a systematic review of clinical effectiveness in adults. Vaccine 2002 ; 20: 2166-73.

14.- Mangtani P, Cutts F, Hall A. Efficacy of polysaccharide pneumococcal vaccine in adults in more developed countries: the state of the evidence. Lancet Infect Dis 2003; 3: 71-8.

15.- Melegaro A, Edmunds W J. The 23-valent pneumococcal polysaccharide vaccine. Part 1. Efficacy of the PPV in the elderly: a comparison of meta-analyses. Eur J Epidemiol 2004; 19 : 353-63.

16.- Huss A, Scott P, Stuck A, Trotter C, Egger M. Efficacy of pneumococcal vaccination in adults: a meta-analysis. Can Med Assoc J 2009; 180 (1): 48-58.

17.- Conaty S, Watson L, Dinnes J, Waugh N. The effectiveness of pneumococcal polysaccharide vaccines in adults: a systematic review of observational studies and comparison with results from randomized controlled trials. Vaccine 2004; 22; 3214-24.

18.- Dear K B G, Andrews R R, Holden J, Ttatham DP. Vaccines for preventing pneumococcal infection in adults. The Cochrane Database Systematic Reviews 2003, Issue 4. Art. No.: CD000422. DOI: 10.1002/14651858. CDC000422.

19.- Kyaw M H, Rose C E Jr, Fry A M, Singleton J A, Moore Z, Zell E R, et al. The influence of chronic illnesses on the incidence of invasive pneumococcal disease in adults. J Infect Dis 2005; 192: 377-86.

20.- Jackson L, Janoff E. Pneumococcal vaccination of elderly adults: new paradigms for protection. Clin Infect Dis 2008; 47: 1328-38.

21.- Skull A S, Andrews R M, Byrnes G B, Kelly H A, Nolan T M, Brown G V. Prevention of community-acquired pneumonia among a cohort of hospitalized elderly: benefit due to influenza and pneumococcal vaccination not demonstrated. Vaccine 2007; 25: 4631-40.

22.- Moberley S A, Holden J, Tatham D P, Andrews R M. Vaccines for preventing pneumoccocal infection in adults. Cochrane Database Syst Rev 2008 Jan 23; (1): CD000422 Disponible en http://www.update-software.com

23.- WHO position Paper. Pneumococcal vaccines. Wkly Epidemiol Rec No. 14, 4 April 2003, pp. 110-9.

24.- Simberkoff M, Cross A, Al-Ibrahim M,
Baltch A, Geiseler P, Nadler J. Efficacy of pneumococcal vaccine in high-risk patients. N Engl J Med 1986; 315: 1318-27.

25.- Davis A L, Aranda C P, Schiffman G, Christianson L C. Pneumococcal infection and immunologic response to pneumococcal vaccine in chronic obstructive pulmonary disease. A pilot study. Chest 1987; 92: 204-12.

26.- Kaufman P. Pneumonia in old age: active immunization against pneumonia with pneumococcus polysaccharide; results of a six year study. Arch Intern Med 1947; 79: 518-31.

27.- Jackson L. Effectiveness of pneumococcal polysaccharide vaccine in older adults. $\mathrm{N}$ Engl $\mathrm{J}$ Med 2003; 348: 1747-55.

28.- Vila-Corcoles A, Ochoa-Gondar O, Llor C, Hospital I, Rodríguez T, Gómez A. Protective effect of pneumococcal vaccine against death by pneumonia in elderly subjects. Eur Respir J 2005; 26 (6): 1086-91.

29.- Vila-Corcoles A, Ochoa-Gondar O, Hospital I, Ansa X, Vilanova A, Rodríguez T, et al. EVAN Study Group. Protective effect of the 23-valent pneumococcal polysaccaharide vaccine in the elderly population: The EVAN-65 Study. Clin Infect Dis 2006; 43: 860-8.

30.- Mooney J J, Weir A, McMenamin J, Ritchie L, Macfarlane T, Simpson C, et al. The impact and effectiveness of pneumoccocal vaccination in Scotland for those aged 65 and over during winter 2003/2004. BMC Inf Dis 2008, 8: 53.

31.- Spindler C, Hedlund J, Jasir A, Henriques B, Orqtvist A. Effects of a large-scale introduction of the polysaccharide vaccine among elderly persons in Stockholm, Sweden. Vaccine 2008; 26: 5541-6.

32.- Go E S, Ballas Z K. Anti-pneumoccocal antibody response in normal subjects: a meta-analysis. J Allergy Clin Immunol 1996; 98: 205-15.

33.- Lexau C, Lynfield R, Danila R, Pilishvili T, Facklam R, Farley M, et al. Changing epidemiology of invasive pneumococcal disease among older adults in the era of pediatric pneumococcal conjugate vaccine. JAMA 2005; 294: 2043-51.

34.- Haber M, Barskey A, Baughman W, Barker L, Whitney C, Shaw K, et al. Herd immunity and pneumococcal vaccine: A quantitative model. Vaccine 2007; 25: 5390-8.

35.- McBean A M, Park Y T, Caldwell D, Yu X. Declining invasive pneumococcal diseases in the U.S. elderly. Vaccine 2005 (23): 5641-5. 